Review Article

\title{
The Therapeutic Potential of the Stem Cell Secretome for Spinal Cord Repair: A Systematic Review and Meta-Analysis
}

Catriona J Cunningham * , Marc Vives Enrich, Molly M Pickford, William Maclntosh-Smith, Wenlong Huang

Institute of Medical Sciences, School of Medicine, Medical Sciences \& Nutrition, University of Aberdeen, Aberdeen AB25 2ZD, United Kingdom; E-Mails: catriona.cunningham@abdn.ac.uk, m.vivesenrich.19@abdn.ac.uk, molly.pickford.16@abdn.ac.uk,w.macintosh-smith.19@abdn.ac.uk, w.huang@abdn.ac.uk

* Correspondence: Catriona Cunningham; E-Mail: catriona.cunningham@abdn.ac.uk

Academic Editor: Ping K Yip

Special Issue: Neuroprotection, Neuroregeneration and Neuroplasticity

OBM Neurobiology

2020, volume 4 , issue 4

doi:10.21926/obm.neurobiol.2004080
Received: October 26, 2020

Accepted: December 06, 2020

Published: December 14, 2020

\begin{abstract}
There is currently no effective treatment for spinal cord injury leaving around $90 \%$ of patients with permanent disabilities. Stem cell therapies are showing promise in preclinical studies of central nervous system injury and there is increasing evidence suggesting the improvements in functional recovery are mediated by paracrine actions. In this systematic review and meta-analysis, we aimed to determine the overall efficacy of stem cell secretome therapies in promoting recovery in preclinical models of spinal cord injury. We searched PubMed and Embase to identify relevant studies. A random effects meta-analysis was conducted using the restricted maximum likelihood estimator. We assessed risk of bias using a modified CAMARADES checklist. Publication bias was then assessed using funnel plots and trim-and-fill analysis. We identified 26 studies that met our inclusion criteria. Overall, stem cell secretome therapies conferred improvement in locomotor score (SMD: $2.30,95 \% \mathrm{Cl}$ : 1.68-2.91), reduction in lesion size (SMD: $3.27,95 \% \mathrm{Cl}: 2.06-4.48)$ and increased axonal
\end{abstract}

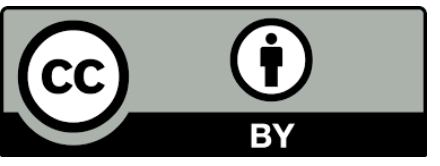

(C) 2020 by the author. This is an open access article distributed under the conditions of the Creative Commons by Attribution License, which permits unrestricted use, distribution, and reproduction in any medium or format, provided the original work is correctly cited. 
profiles in the lesion (SMD: 2.36, 95\% Cl: 1.02-3.71). We found there was significant asymmetry in the funnel plots for all three outcome measures, suggesting publication bias. Trim-and-fill analysis estimated 19 and 3 unpublished studies in the locomotor score and axonal profiles datasets respectively. The median score on the modified CAMARADES checklist was 4 (IQR 4-5). Reporting of power calculations and allocation concealment was absent. The stem cell secretome is showing great potential as a therapy for spinal cord injury. As the vast majority of studies began treatment acutely and favoured reduction in lesion size, we argue neuroprotection is likely the key mechanism of action. Future studies should focus on exploring the contribution of other mechanisms, the mediators involved and effect of treatment at a chronic stage of injury.

\section{Keywords}

Stem cells; cell therapy; spinal cord injury; neuroprotection; regeneration; systematic review; meta-analysis

\section{Introduction}

Spinal cord injury $(\mathrm{SCl})$ was described as an "ailment not to be treated" in the Edwin Smith surgical papyrus in $1700 \mathrm{BC}$ [1] and almost 4,000 years later, there is still no cure. Of the 27 million patients worldwide living with $\mathrm{SCl}$ [2], around 90\% experience long-term disabilities including loss of motor and sensory functions below the injury level. There are a number of major obstacles to $\mathrm{SCl}$ repair including the limited intrinsic regenerative capacity of the adult mammalian central nervous system (CNS) neurons, physical barrier of a cystic cavity and presence of numerous inhibitory molecules at the injury site including chondroitin sulphate proteoglycans (CSPGs) which prevent axon regrowth [3].

In the past decade, there has been great interest in the development of regenerative medicine and tissue engineering approaches for $\mathrm{SCl}$ repair. Stem cell therapies, in particular, are showing great promise. Several cell types have been progressed to clinical trial including neural stem cells (NSCs), bone marrow aspirate and mesenchymal stem cells (MSCs), the latter of which is the most widely investigated [4]. There is increasing evidence that the improvements in functional recovery observed in CNS injury models following stem cell transplantation are mediated by paracrine actions [5]. The secretome is a collective term for the vast array of secreted chemokines, cytokines, growth factors and extracellular vesicles (EVs) [6]. Numerous studies have characterised the stem cell secretome through techniques including mass spectroscopy and bioinformatics. Growth factors including brain-derived neurotrophic factor (BDNF), nerve growth factor (NGF), vascular endothelial growth factor (VEGF), basic fibroblast growth factor (bFGF) and epidermal growth factor (EGF) have been detected in both the MSC and NSC secretome [7, 8]. However, it must be noted that there is great heterogeneity between donors, tissue source and cell types.

In more recent years, many groups have been focusing specifically on the role of EVs in the stem cell secretome. EVs are membrane-bound vesicles which play an important role in intracellular signalling [9]. EVs can be characterised based on their biogenesis: apoptotic bodies $(500-4,000 \mathrm{~nm})$ arise as a result of plasma membrane blebbing and cell disintegration during 
apoptosis; microvesicles (50-2,000 nm) bud directly from the membrane whereas exosomes (30$100 \mathrm{~nm}$ ) are released when an multivesicular body fuses with the membrane [9, 10]. While EVs can contain proteins and lipids, most research into therapies for CNS repair has focussed on the mRNA and microRNA (miRNA) cargo [11]. For example, EVs derived from MSCs overexpressing miR-133b or the miR-17/92 cluster has previously been associated with improvements in recovery in rodent models of stroke [12-14].

Acellular secretome therapies hold great translational potential and have several advantages over conventional cell therapies including the mitigation of the risk of immune rejection, reduced risk of tumourigenesis and ability to cryopreserve treatments without needing to consider the issues of maintaining cell viability [6]. This systematic review and meta-analysis aims to determine the overall efficacy of stem cell secretome therapies in promoting locomotor recovery, lesion volume reduction and axonal regrowth in preclinical models of $\mathrm{SCl}$. We also hope to identify possible sources of bias and highlight future avenues of research.

\section{Materials and Methods}

This systematic review followed the Preferred Reporting Items for Systematic Reviews and Meta-Analyses statement (PRISMA) guidelines [15]. The PRISMA checklist includes a 27 item list defined by a panel of experts as the minimum reporting criteria for systematic reviews and metaanalyses. We preregistered a protocol in the PROSPERO database (CRD42020167718).

\subsection{Search Strategy}

We searched PubMed and Embase (OVID) for articles published in English from January 2008 onwards using the search strategy detailed in the PROSPERO protocol. Previously published filters were used to limit searches to animal studies only $[16,17]$. We also screened relevant review articles for additional studies. The last search was performed on 23 January 2020.

\subsection{Study Selection}

After removing duplicates, we first screened the titles and abstracts of articles for eligibility. Articles which were clearly irrelevant (e.g. reviews, irrelevant disease model) were excluded. In the second screen, the full texts of identified articles were screened for against complete inclusion criteria. Studies assessing the therapeutic potential of stem cell secretome therapies in pre-clinical models of $\mathrm{SCl}$ were included. We included studies if a locomotor score was used as an outcome measure and there was an appropriate control group ( $\mathrm{SCl}+$ vehicle/control). Studies with sham surgery or naïve control groups only were excluded. Two independent reviewers (MP, MEV) conducted the screening and disagreements were resolved by discussion with a third reviewer (CC).

\subsection{Data Extraction}

Two independent reviewers (MP, MEV) extracted qualitative data from the included articles. Any discrepancies which occurred were resolved by a third reviewer (CC). We extracted study design information including the following: species; strain; $\mathrm{SCl}$ model and injury level; stem cell secretome therapy including cell source; route of administration and behavioural tests used. Study 
quality was then assessed using an adapted 7-point CAMARADES (Collaborative Approach to Meta-Analysis and Review of Animal Data in Experimental Studies) Risk of Bias Checklist [18]. The items were: 1) peer reviewed publication 2) random allocation to group 3) allocation concealment 4) blinded assessment of outcome 5) sample size calculation/power calculation 6) compliance with animal welfare regulations 7) statement of potential conflict of interest.

Two independent reviewers (MEV, WMS) extracted locomotor data from the included studies. Data from the secondary outcome measures was then extracted by a second team of reviewers (CC, WMS). We defined locomotor score (any scale) as the primary outcome measure and the secondary outcome measures as lesion size and axonal regrowth. We extracted mean values and SEM or SD from the article text where possible. Where one control group was used for multiple treatment groups, we corrected for this by dividing the number of animals in the control group by the number of treatment groups. In instances where outcome measures were assessed at multiple timepoints, data from the last timepoint were extracted. Where data were only presented graphically, we used the online graphical tool WebPlotDigitizer (https://automeris.io/WebPlotDigitizer/) to extract values from the graphs. Estimates were crosschecked by a second independent reviewer and where these varied by $<10 \%$, means were taken. Any differences $>10 \%$ were resolved through discussion. Where it was not possible to extract data using this tool and exact $n$ numbers were not reported, we emailed authors for clarification. If the data were not made available after two attempts, we excluded the corresponding studies from the meta-analysis.

\subsection{Statistical Analysis}

We used the metafor package [19] in RStudio V1.3.959 (RStudio, USA), R version 4.0.1 for all statistical analyses and graphs. Standardised mean difference (SMD) effect sizes were calculated using Hedges' $g$. For all outcome measures, a positive SMD favours treatment. A random effects meta-analysis was conducted using the restricted maximum likelihood estimator for all outcome measures due to the high heterogeneity in the data. We used funnel plots to visualise publication bias and confirmed by Egger's regression test. Trim-and-fill analysis was used to estimate the number of "missing" unpublished studies and calculate an adjusted effect size accounting for publication bias. Heterogeneity was assessed using $l^{2}$ (between-study variance not attributed to sampling error) and $\mathrm{Tau}^{2}$ (between-study variance). We performed subgroup analyses to explore sources of heterogeneity in the data including use of reporting of blinding, stem cell type and route of administration. Independent random effects models were fitted to subgroups and estimates were compared with a Wald-type test. Analysis was only performed when there were at least 4 comparisons in a subgroup [20]. Significance was defined as $p<0.05$.

\section{Results}

\subsection{Study Characteristics}

We identified a total of 919 articles, of which 26 met our inclusion criteria (Figure 1). Study details including secretome intervention, timepoint of administration and $\mathrm{SCl}$ model are summarised in Table S1. The vast majority of included studies were conducted in rats $(n=21)$ with the remainder using mice $(n=5)$. All studies induced traumatic SCl at the thoracic level $(T 7-12)$ 
using the following models: contusion ( $n=15)$; compression $(n=7)$; hemisection $(n=3)$ and complete transection $(n=1)$. MSCs were by far the most common stem cell type used $(n=19)$ with remaining studies using NSCs $(n=4), \operatorname{OECs}(n=1), \operatorname{ESCs}(n=1)$ and stem cells derived from breast milk $(n=1)$. There was close to an even division between studies which administered conditioned medium $(n=13)$ and EVs $(n=12)$. Kim et al. [21] administered exosome-mimetic nanovesicles derived from human MSCs which encapsulated iron oxide nanoparticles to facilitate magnet-guided navigation to the lesion. Only one used a combination therapy which was the use of a collagen-based hydrogel as a drug delivery system for human deciduous dental pulp MSC-derived conditioned medium [22]. There were three studies which used genetically modified stem cells to overexpress MiR-126 [23], MiR-113b [24] and VEGF-A [25]. The most widely used route of administration used was IV $(n=14)$ followed by intrathecal $(n=8)$, IP $(n=3)$ and intralesional $(n=1)$.

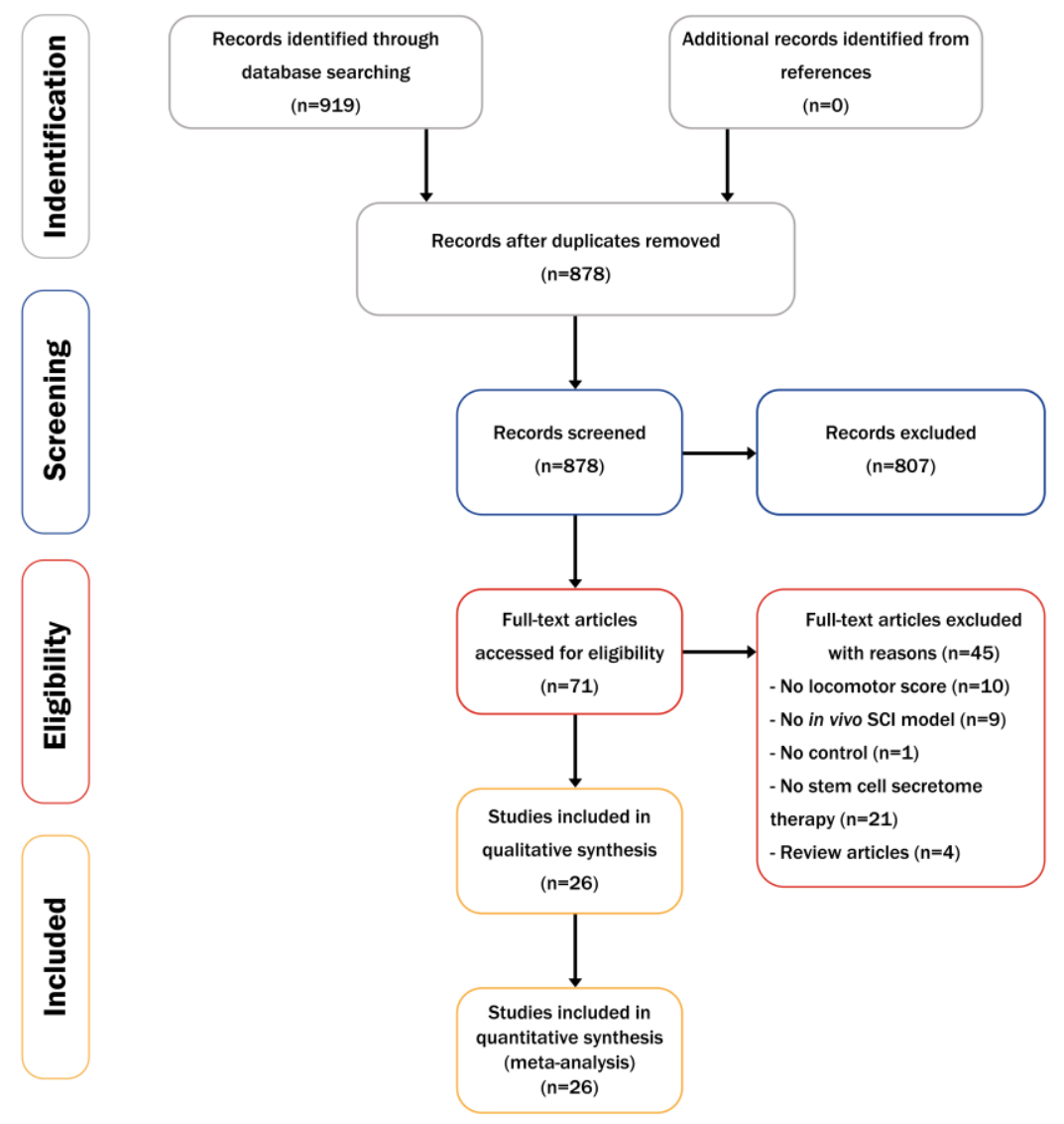

Figure 1 PRISMA flow chart summarising the literature search and number of included studies.

There was great variability in the dosing strategies in the included studies. Single dose $(n=14)$, multiple dosing $(n=9)$, continuous infusion $(n=3)$ of secretome therapies were used. However, the majority of studies did begin treatment acutely within $3 \mathrm{~h}$ of injury onset $(n=22)$. Only one study began treatment at greater than $48 \mathrm{~h}$. Chudickova et al. [26] administered doses of conditioned medium derived from human MSCs at 1, 2 and 3 weeks post-injury.

Liang et al. [27] used biotinylated dextran amine (BDA) tracing to study axonal regeneration. All other studies used markers such as GAP-43 or class III $\beta$-tubulin to label axons in the lesion then 
quantified by area of positive staining or counts. We aimed to evaluate axonal regrowth as a secondary outcome measure but this was not feasible as these methods do not distinguish between axonal regeneration, sprouting and white matter sparing. We proceeded with data extraction as we felt it was still of interest and have instead termed the outcome measure as axonal profiles in the lesion.

\subsection{Synthesised Results}

All 26 studies (35 comparisons) were assessed in our meta-analysis of locomotor score. We identified 20 and 12 comparisons for our secondary outcome measures lesion size and axonal profiles respectively. All 4 authors contacted for clarification of their data responded. Overall, stem cell secretome therapies improved locomotor scores compared with control (SMD: 2.30, 95\% confidence interval $\left.[\mathrm{Cl}]: 1.68-2.91, p<0.0001, \mathrm{Tau}^{2}=2.7, l^{2}=86.7 \%\right)$. This equates to a $\sim 68.9 \%$ improvement in locomotor score. In similarity, treatment favoured improvements in the secondary outcome measures leading to around a 32.6\% reduction in lesion size (SMD: 3.27, 95\% $\mathrm{Cl}$ : 2.06-4.48, $\left.p<0.0001, \mathrm{Tau}^{2}=6.17, I^{2}=90.3 \%\right)$ and $\sim 177 \%$ increase in axonal profiles in the lesion (SMD: 2.36, 95\% Cl: 1.02-3.71, $p<0.0001, \mathrm{Tau}^{2}=4.46, l^{2}=89.7 \%$ ).

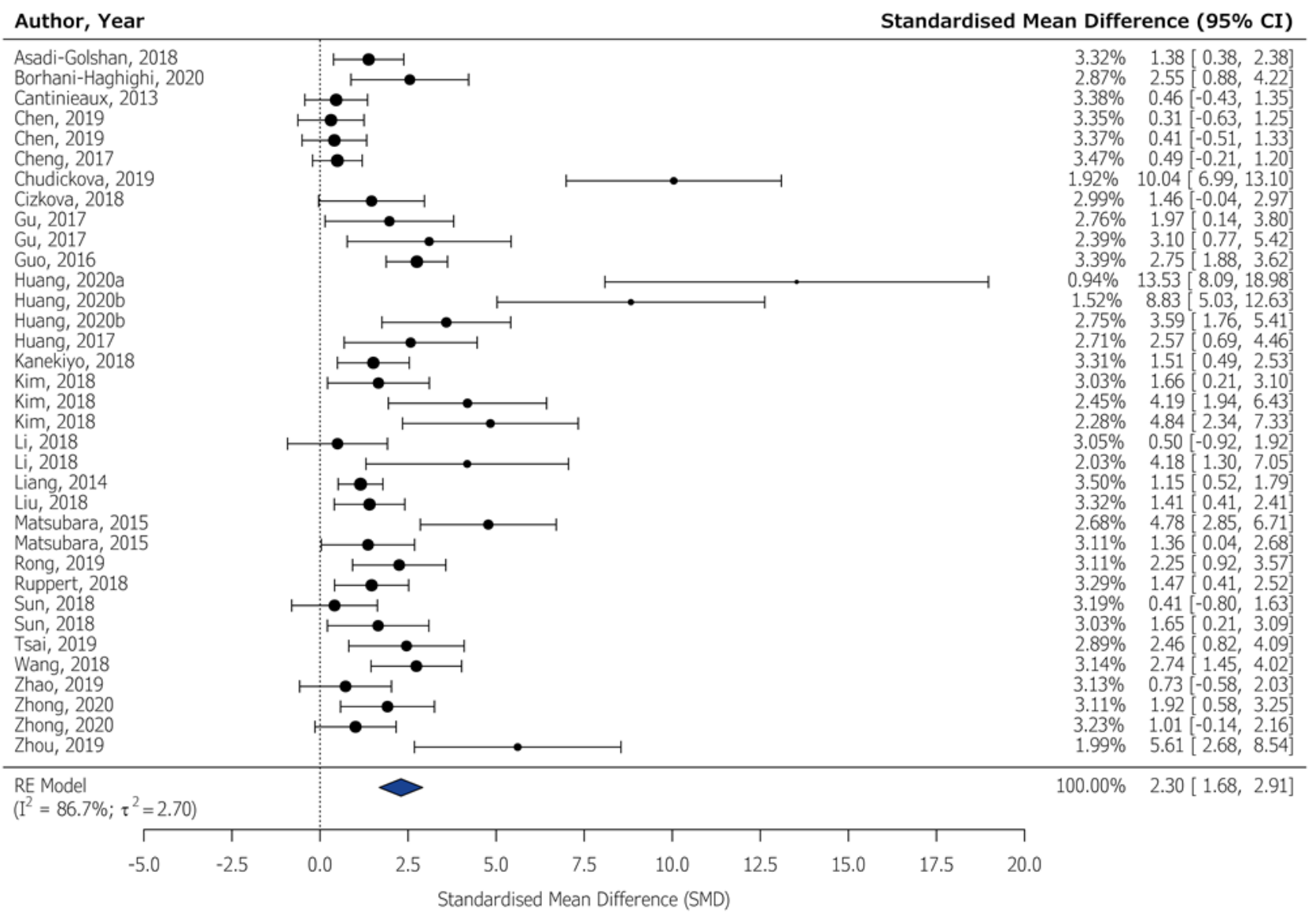

Figure 2 Forest plot showing effect of stem cell secretome therapies on locomotor score. A positive SMD represents an improvement in locomotor score. Points indicate effect size estimates for each individual comparison and error bars are $95 \%$ confidence intervals (Cls). Point size indicates the relative weight of each estimate. Diamond represents overall effect size and diamond width is $95 \% \mathrm{Cl}$. 
A

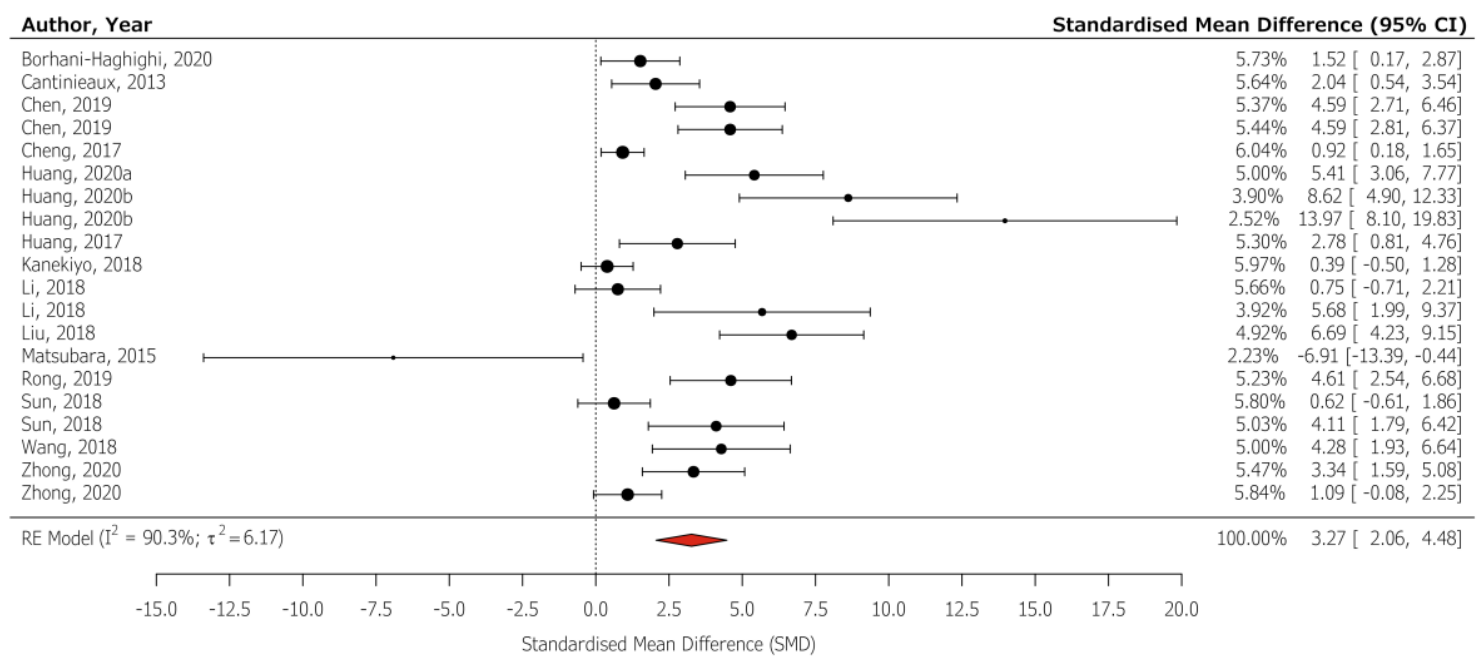

B

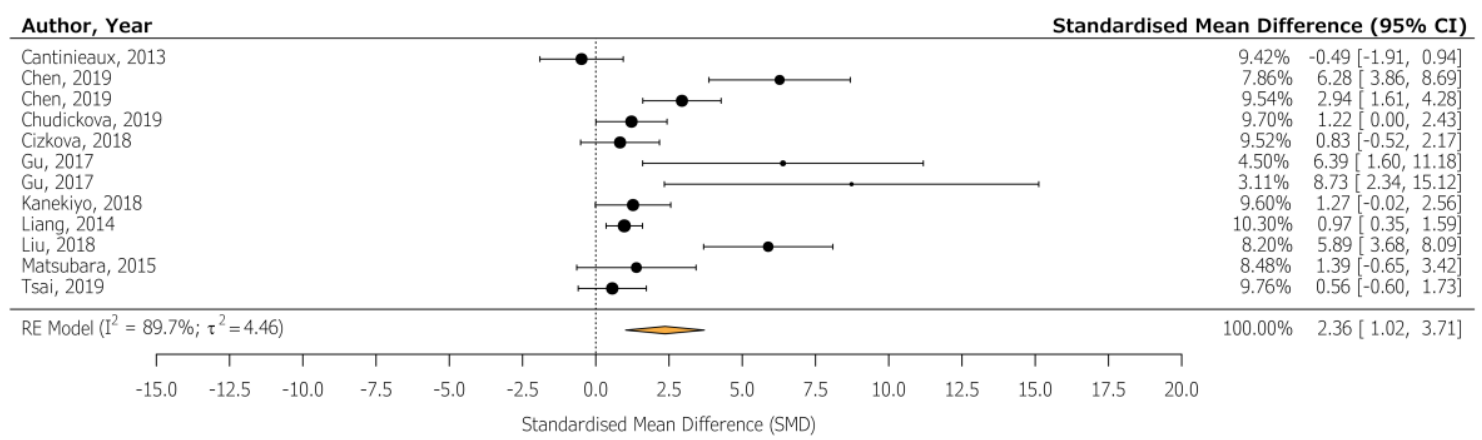

Figure 3 Forest plots showing effect of stem cell secretome therapies on secondary outcome measures lesion size (A) and axonal profiles in the lesion (B). Positive SMDs demonstrate a reduction in lesion volume and increased axonal profiles. Points indicate effect size estimates for each individual comparison and error bars are $95 \%$ confidence intervals (Cls). Point size indicates the relative weight of each estimate. Diamond represents overall effect size and diamond width is $95 \% \mathrm{Cl}$.

To explore sources of heterogeneity in the data, we then conducted a subgroup analysis. In the locomotor score data, secretome therapies derived from NSCs were suggested to be less effective. The effect size of NSCs was $1.20(0.620-1.78)$ compared with $2.65(1.73-3.58, p=0.009$, Table S2) for MSCs and 2.64 (1.96-3.32, $p=0.009$, Table S2) for the remaining other stem cell types. EV therapies were associated with a greater reduction in lesion size than conditioned medium (SMD: $1.8195 \% \mathrm{Cl}: 0.179-3.44$ vs. SMD: $4.18,95 \% \mathrm{Cl}: 2.62-5.75, p=0.039$, Table S3).

\subsection{Risk of Bias}

We assessed risk of bias using a modified 7-point CAMARADES checklist. As shown in Table 1, the median score was 4 (IQR 4-5). While the majority of studies reported blinding to outcome assessment $(77.8 \%)$ and randomisation $(70.4 \%)$, there were no studies which included a power calculation or reported allocation concealment. 
Table 1 Risk of bias as assessed using a modified CAMARADES checklist.

\begin{tabular}{ll}
\hline Checklist Item & \\
\hline 1. Peer reviewed (\%) & 100 \\
2. Random allocation to group (\%) & 70.4 \\
3. Allocation concealment (\%) & 0 \\
4. Blinded assessment outcome (\%) & 77.8 \\
5. Sample size calculation (\%) & 0 \\
6. Animal welfare regulations (\%) & 92.6 \\
7. Conflict of interest (\%) & 92.6 \\
Median study quality (IQR) & $4(4-5)$ \\
\hline
\end{tabular}

Leading on from this, we then assessed publication bias. As shown in Figure 4A, there was pronounced funnel plot asymmetry for locomotor score data indicating there was publication bias and this was confirmed by Egger's regression ( $p>0.001$ ). Trim-and-fill analysis (Figure 4B) estimated there were 19 "missing" unpublished studies on the left-hand side of the funnel plot with neutral effect sizes. When adjusted for, this reduced effect size from 2.30 to 0.94 . While there was also asymmetry in the funnel plot for the lesion size data (Figure 4C; $p>0.001$ ), trimand-fill analysis did not estimate any unpublished studies (Figure 4C). Furthermore, there was pronounced asymmetry in the funnel plot of the axonal profiles data (Figure 4E) as indicated by Egger's regression $(p=0.0002$ ). Trim-and-fill analysis predicted there were 3 "missing" studies which when accounted for, reduced effect size from 3.71 to 1.55 . 
A

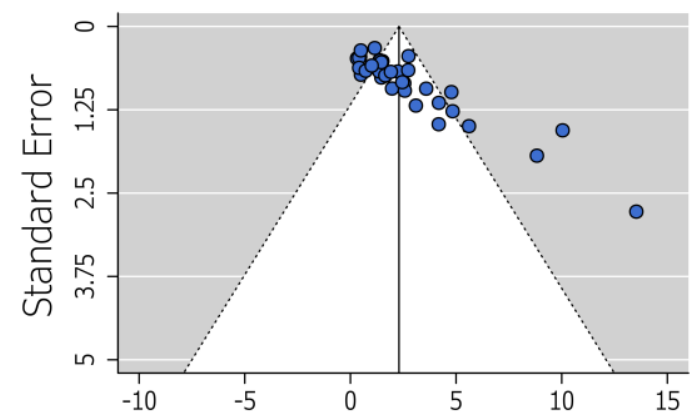

B

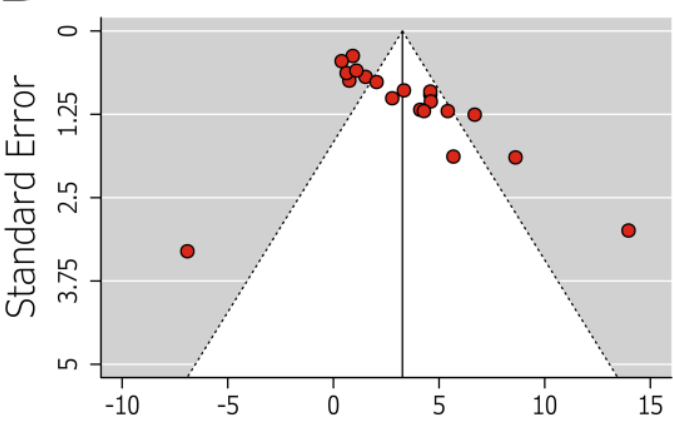

C

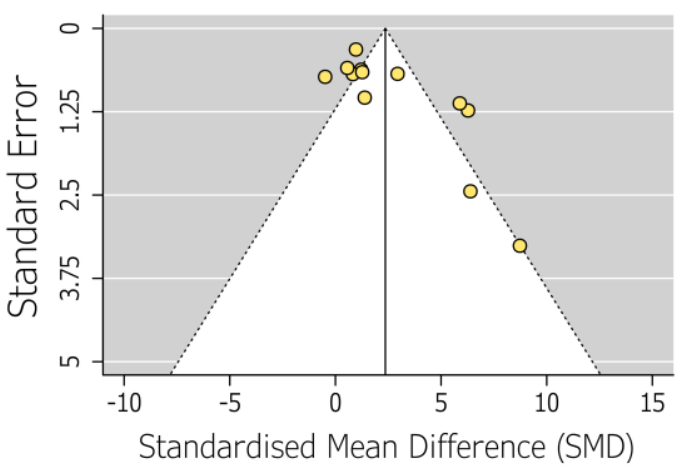

D

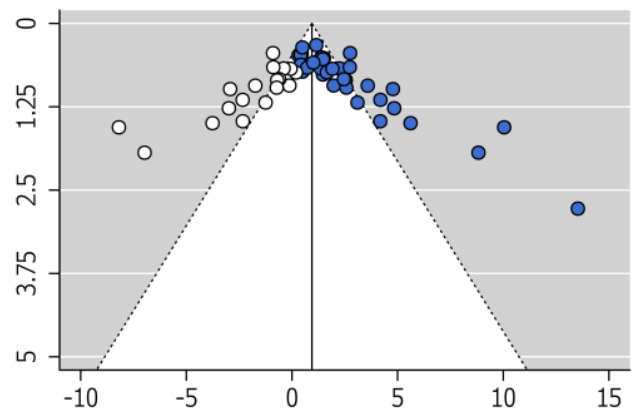

$\mathbf{E}$

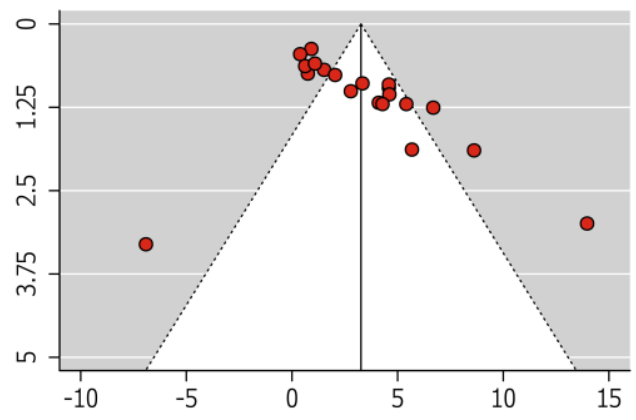

$\mathbf{F}$

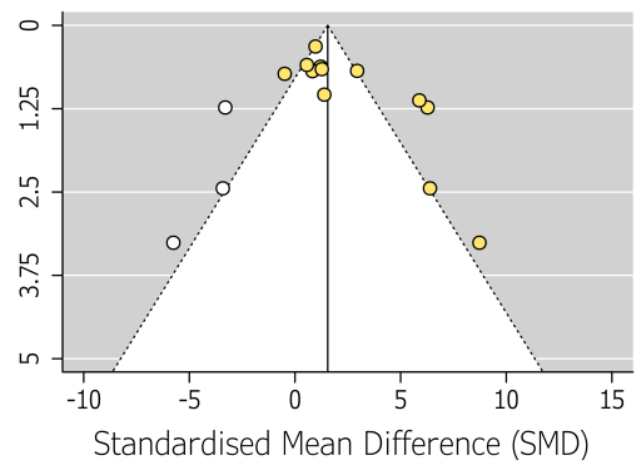

Figure 4 Assessment of publication bias in locomotor score data. Funnel plots show pronounced asymmetry in locomotor score (A), lesion size (B) and axonal profiles (C). Vertical lines indicate the effect size. Trim-and-fill analysis of the locomotor score (D), lesion size (E) and axonal profiles in the lesion (F) datasets predicted 19, 0 and 3 "missing" studies (unfilled circles) respectively. White funnels show $95 \% \mathrm{Cls.}$

\section{Discussion}

In this systematic review and meta-analysis of the efficacy of stem cell secretome therapies in preclinical models of $\mathrm{SCl}$, we identified 26 studies that met our inclusion criteria. Overall, treatment favoured improvement in locomotor score, reduction in lesion size and increased presence of axonal profiles in the lesion. We assessed risk of bias using a modified CAMARADES checklist finding that although reporting of blinding and randomisation was high, no studies reported allocation concealment and power calculations. We found there was significant 
asymmetry in the funnel plots for all three outcome measures indicating publication bias. Leading on from this, we conducted trim-and-fill analysis identified which estimated there were 19, 0 and 3 unpublished studies for the locomotor score, lesion size and axonal profiles datasets respectively.

While there was no consensus on whether single, multiple doses or continuous infusion of stem cell secretome was optimal, almost all studies began treatment acutely within $24 \mathrm{~h}$ of injury onset. This is within the time window for $\mathrm{SCl}$ neuroprotection and, indeed, our results showed that treatment was associated with a reduction in lesion size. We therefore argue that neuroprotection is likely the key mechanism of action which contributed to the observed improvements in locomotor recovery. At present, the exact mechanisms of action of the stem cell secretome are unclear and this should be the focus of future studies. We also recommend that treatment at subacute and chronic timepoints after $\mathrm{SCl}$ be investigated further and this would be useful for distinguishing between neuroprotection and other mechanisms. Given the vast array of cytokines, chemokines, growth factors and EVs present in the secretome, it is likely that a combination of mechanisms and mediators are involved [5]. The MSC secretome in particular, contains a number of molecules which may have immunomodulatory effects on immune cells such as activated microglia and infiltrated macrophages after $\mathrm{SCl}$. For example, secretion of prostaglandin E2 from MSCs has been shown to drive macrophages towards a less pro-inflammatory phenotype in a mouse model of sepsis [28]. A number of studies included in our meta-analysis reported that stem cell secretome therapies polarised macrophages towards a more regulatory M2-like phenotype $[21,29,30]$. Angiogenesis may also contribute to observed improvements in recovery. As previously mentioned, both MSCs and NSCs secrete VEGF which is a potent promoter of angiogenesis. One included study showed that administration of NSC-derived exosomes transfected VEGF-A significantly enhanced angiogenesis and locomotor recovery, compared with exosomes in which VEGF-A was knocked down [25].

The holy grail in $\mathrm{SCl}$ research is identifying a therapy capable of promoting axonal regeneration. However, a lack of consistency in the use of the terms regeneration, sprouting and growth in the literature has previously been described [31], which could lead to the misinterpretation of results. We encountered this as a potential issue with the studies included in our meta-analysis. Many studies reported that their stem cell secretome therapies promoted axonal regeneration or sprouting. As only one study used tract tracing methods, these conclusions were not be substantiated. Given that treatment appeared to promote $\mathrm{SCl}$ neuroprotection, the observed increased axon staining in the lesion could be attributed to white matter sparing rather than sprouting or regeneration.

To address the issues of poor study reporting and transparency in animal research, The National Centre for the Replacement, Refinement and Reduction of Animals (NC3Rs) developed the Animal Research: Reporting of in vivo Experiments (ARRIVE) guidelines [32]. Since publication in 2010, many journals have endorsed these guidelines and now require authors to complete an ARRIVE checklist alongside their submission. However, a recent study of manuscripts submitted to PLOS ONE [33] showed that this was not sufficient to ensure compliance. It is therefore not surprising that while all of the studies included in our systematic review were published after the ARRIVE guidelines, reporting of power calculations and allocation concealment was absent. A major issue decreasing the reliability of results is a lack of power in animal experiments. Button et al. [34] estimated that the median statistical power of neuroscience studies was $21 \%$. It is likely that many of the studies included in our meta-analysis were therefore underpowered and this 
may have resulted in our reported effect sizes being overestimated. Publication bias is another well-documented issue in animal research, whereby studies with negative or neutral findings are far less likely to be published. In an analysis of preclinical stroke studies, Sena et al. [35] found that just $2.2 \%$ of studies did not report significant results and publication bias may have inflated efficacy by a third. As our meta-analysis indicated there was publication bias for all three of our outcomes, this may have led to an overestimation in our reported effect sizes.

\section{Conclusions}

Our systematic review and meta-analysis showed the stem cell secretome may have great potential as a therapy for spinal cord injury. As the vast majority of studies began treatment acutely and lesion volume was reduced, we argue neuroprotection is the key mechanism of action. An important but challenging step in the translation of stem cell secretome therapies to the clinic will be to identify the exact mechanisms of action and the mediators involved.

\section{Acknowledgments}

We would like to thank Drs Ya-Tzu Chen (Taipei Veterans General Hospital), May-Jywan Tsai (Taipei Veterans General Hospital), Kenji Kanekiyo (Aino University) and Yi Ren (Florida State University) for responding to our requests for clarification on their studies. We would also like to thank Dr Jack Rivers-Auty (University of Tasmania) for his advice on statistical analysis.

\section{Additional Materials (if any)}

The following additional materials are uploaded at the page of this paper.

1. Table S1: Summary of the study design of all publications included in the systematic review

2. Table S2: Subgroup analysis of locomotor score data

3. Table S3: Subgroup analysis of lesion size data

4. Table S4: Subgroup analysis of axonal profiles data

5. Raw data file

\section{Author Contributions}

Catriona Cunningham designed the study, extracted data, conducted data analysis and wrote the manuscript. Marc Vives-Enrich and Molly Pickford performed literature searches, screening and data extraction. William Maclntosh-Smith extracted data. Wenlong Huang provided supervision and edited the manuscript.

\section{Funding}

This work was supported by Graham and Pam Dixon and the Scottish Rugby Union.

\section{Competing Interests}

The authors have declared that no competing interests exist. 


\section{References}

1. Donovan WH. Donald Munro Lecture. Spinal cord injury-past, present, and future. J Spinal Cord Med. 2007; 30: 85-100.

2. James SL, Bannick MS, Montjoy-Venning WC, Lucchesi LR, Dandona L, Dandona R, et al. Global, regional, and national burden of traumatic brain injury and spinal cord injury, 1990-2016: A systematic analysis for the Global Burden of Disease Study 2016. Lancet Neurol. 2019; 18: 5687.

3. Fawcett JW. The struggle to make CNS axons regenerate: Why has it been so difficult? Neurochem Res. 2019; 45: 144-158.

4. Willison AG, Smith S, Davies BM, Kotter MR, Barnett SC. A scoping review of trials for cellbased therapies in human spinal cord injury. Spinal Cord. 2020; 58: 844-856.

5. Pinho AG, Cibrão JR, Silva NA, Monteiro S, Salgado AJ. Cell secretome: Basic insights and therapeutic opportunities for CNS disorders. Pharmaceuticals. 2020; 13: 31.

6. Cunningham CJ, Redondo-castro E, Allan SM. The therapeutic potential of the mesenchymal stem cell secretome in ischaemic stroke. J Cereb Blood Flow Metab. 2018; 38: 1276-1292.

7. Ferreira JR, Teixeira GQ, Santos SG, Barbosa MA, Almeida-Porada G, Gonçalves RM. Mesenchymal stromal cell secretome: Influencing therapeutic potential by cellular preconditioning. Front Immunol. 2018; 9: 2837.

8. Willis MC, Nicaise AM, Peruzzotti-Jametti L, Pluchino $S$. The neural stem cell secretome and its role in brain repair. Brain Res. 2020; 1729: 146615.

9. Raposo G, Stoorvogel W. Extracellular vesicles: Exosomes, microvesicles, and friends. J Cell Biol. 2013; 200: 373-383.

10. Akers JC, Gonda D, Kim R, Carter BS, Chen CC. Biogenesis of extracellular vesicles (EV): Exosomes, microvesicles, retrovirus-like vesicles, and apoptotic bodies. J Neurooncol. 2013; 113: 1-11.

11. Drago D, Cossetti C, Iraci N, Gaude E, Musco G, Bachi A, et al. The stem cell secretome and its role in brain repair. Biochimie. 2013; 95: 2271-2285.

12. Shen H, Yao X, Li H, Li X, Zhang T, Sun Q, et al. Role of exosomes derived from mir-133b modified mscs in an experimental rat model of intracerebral hemorrhage. J Mol Neurosci. 2018; 64: 421-430.

13. Xin H, Katakowski M, Wang FJ, Qian JY, Liu XS, Ali MM, et al. MicroRNA-17-92 cluster in exosomes enhance neuroplasticity and functional recovery after stroke in rats. Stroke. 2017; 48: 747-753.

14. Xin H, Li Y, Liu Z, Wang X, Shang X, Cui Y, et al. MiR-133b promotes neural plasticity and functional recovery after treatment of stroke with multipotent mesenchymal stromal cells in rats via transfer of exosome-enriched extracellular particles. Stem Cells. 2013; 31: 2737-2746.

15. Moher D, Liberati A, Tetzlaff J, Altman DG, PRISMA Group. Preferred reporting items for systematic reviews and meta-analyses: The PRISMA statement. BMJ. 2009; 6: e1000097.

16. Hooijmans $C R$, Tillema $A$, Leenaars $M$, Ritskes-Hoitinga $M$. Enhancing search efficiency by means of a search filter for finding all studies on animal experimentation in PubMed. Lab Anim. 2010; 44: 170-175.

17. de Vries RB, Hooijmans CR, Tillema A, Leenaars M, Ritskes-Hoitinga M. Updated version of the Embase search filter for animal studies. Lab Anim. 2014; 48: 88. 
18. Macleod MR, O'Collins T, Howells DW, Donnan GA. Pooling of animal experimental data reveals influence of study design and publication bias. Stroke. 2004; 35: 1203-1208.

19. Viechtbauer W. Conducting meta-analyses in $R$ with the metafor package. J Stat Softw. 2010; 36: 1-48.

20. Fu R, Gartlehner G, Grant M, Shamliyan T, Sedrakyan A, Wilt TJ, et al. Conducting quantitative synthesis when comparing medical interventions: AHRQ and the effective health care program [Internet]. Rockville: Agency for Healthcare Research and Quality; 2008. Available: https://www.ncbi.nlm.nih.gov/books/NBK49407/.

21. Kim HY, Kumar H, Jo MJ, Kim J, Yoon JK, Lee JR, et al. Therapeutic efficacy-potentiated and diseased organ-targeting nanovesicles derived from mesenchymal stem cells for spinal cord injury treatment. Nano Lett. 2018; 18: 4965-4975.

22. Asadi-Golshan R, Razban V, Mirzaei E, Rahmanian A, Khajeh S, Mostafavi-Pour Z, et al. Sensory and Motor Behavior Evidences Supporting the Usefulness of Conditioned Medium from Dental Pulp-Derived Stem Cells in Spinal Cord Injury in Rats. Asian Spine J. 2018; 12: 785-793.

23. Huang $\mathrm{JH}, \mathrm{Xu} \mathrm{Y}$, Yin XM, Lin FY. Exosomes derived from miR-126-modified MSCs promote angiogenesis and neurogenesis and attenuate apoptosis after spinal cord injury in rats. Neuroscience. 2020; 424: 133-145.

24. Li D, Zhang P, Yao XY, Li HY, Shen HT, Li X, et al. Exosomes derived from miR-133b-modified mesenchymal stem cells promote recovery after spinal cord injury. Front Neurosci. 2018; 12: 845.

25. Zhong D, Cao Y, Li CJ, Li M, Rong ZJ, Jiang L, et al. Neural stem cell-derived exosomes facilitate spinal cord functional recovery after injury by promoting angiogenesis. Exp Biol Med. 2020; 245: 54-65.

26. Chudickova $M$, Vackova I, Machova Urdzikova L, Jancova $P$, Kekulova $K$, Rehorova $M$, et al. The effect of wharton jelly-derived mesenchymal stromal cells and their conditioned media in the treatment of a rat spinal cord injury. Int J Mol Sci. 2019; 20: 4516.

27. Liang $P$, Liu J, Xiong J, Liu Q, Zhao J, Liang $H$, et al. Neural stem cell-conditioned medium protects neurons and promotes propriospinal neurons relay neural circuit reconnection after spinal cord injury. Cell Transplant. 2014; 23: 45-56.

28. Németh K, Leelahavanichkul A, Yuen PST, Mayer B, Parmelee A, Doi K, et al. Bone marrow stromal cells attenuate sepsis via prostaglandin E 2-dependent reprogramming of host macrophages to increase their interleukin-10 production. Nat Med. 2009; 15: 42-49.

29. Sun GD, Li GQ, Li DH, Huang WJ, Zhang RW, Zhang $H$, et al. hucMSC derived exosomes promote functional recovery in spinal cord injury mice via attenuating inflammation. Mater Sci Eng C Mater Biol Appl. 2018; 89: 194-204.

30. Matsubara K, Matsushita Y, Sakai K, Kano F, Kondo M, Noda M, et al. Secreted ectodomain of sialic acid-binding Ig-like lectin- 9 and monocyte chemoattractant protein-1 promote recovery after rat spinal cord injury by altering macrophage polarity. J Neurosci. 2015; 35: 2452-2464.

31. Tuszynski $\mathrm{MH}$, Steward $\mathrm{O}$. Concepts and methods for the study of axonal regeneration in the CNS. Neuron. 2012; 75: 777-791.

32. Kilkenny C, Browne WJ, Cuthill IC, Emerson M, Altman DG. Improving bioscience research reporting: The ARRIVE guidelines for reporting animal research. PLoS Biol. 2010; 8: e1000412.

33. Hair K, Macleod MR, Sena ES. A randomised controlled trial of an intervention to improve compliance with the ARRIVE guidelines (IICARus). Res Integr Peer Rev. 2019; 4: 12. 
34. Button KS, loannidis JP, Mokrysz C, Nosek BA, Flint J, Robinson ES, et al. Power failure: Why small sample size undermines the reliability of neuroscience. Nat Rev Neurosci. 2013; 14: 365376.

35. Sena ES, van der Worp HB, Bath PM, Howells DW, Macleod MR. Publication bias in reports of animal stroke studies leads to major overstatement of efficacy. PLoS Biol. 2010; 8: e1000344.

36. Borhani-Haghighi M, Navid S, Mohamadi Y. The therapeutic potential of conditioned medium from human breast milk stem cells in treating spinal cord injury. Asian Spine J. 2020; 14: 131138.

37. Cantinieaux D, Quertainmont R, Blacher S, Rossi L, Wanet T, Noel A, et al. Conditioned medium from bone marrow-derived mesenchymal stem cells improves recovery after spinal cord injury in rats: An original strategy to avoid cell transplantation. PLoS One. 2013; 8: e69515.

38. Chen YT, Tsai MJ, Hsieh N, Lo MJ, Lee MJ, Cheng $H$, et al. The superiority of conditioned medium derived from rapidly expanded mesenchymal stem cells for neural repair. Stem Cell Res Ther. 2019; 10: 390.

39. Cheng ZJ, Bosco DB, Sun L, Chen XM, Xu YS, Tai WJ, et al. Neural stem cell-conditioned medium suppresses inflammation and promotes spinal cord injury recovery. Cell Transplant. 2017; 26: 469-482.

40. Cizkova D, Cubinkova V, Smolek T, Murgoci AN, Danko J, Vdoviakova K, et al. Localized intrathecal delivery of mesenchymal stromal cells conditioned medium improves functional recovery in a rat model of spinal cord injury. Int J Mol Sci. 2018; 19: E870.

41. Gu MC, Gao ZC, Li XH, Guo L, Lu T, Li HY, et al. Conditioned medium of olfactory ensheathing cells promotes the functional recovery and axonal regeneration after contusive spinal cord injury. Brain Res. 2017; 1654: 4354.

42. Guo L, Rolfe AJ, Wang X, Tai WJ, Cheng ZJ, Cao K, et al. Rescuing macrophage normal function in spinal cord injury with embryonic stem cell conditioned media. Mol Brain. 2016; 9: 48.

43. Huang JH, Fu C-H, Xu Y, Yin XM, Cao Y, Lin FY. Extracellular vesicles derived from epidural fatmesenchymal stem cells attenuate NLRP3 inflammasome activation and improve functional recovery after spinal cord injury. Neurochem Res. 2020; 45: 760-771.

44. Huang JH, Yin XM, Xu Y, Xu CC, Lin X, Ye FB, et al. Systemic administration of exosomes released from mesenchymal stromal cells attenuates apoptosis, inflammation, and promotes angiogenesis after spinal cord injury in rats. J Neurotrauma. 2017; 34: 3388-3396.

45. Kanekiyo K, Wakabayashi T, Nakano N, Yamada Y, Tamachi M, Suzuki Y, et al. Effects of intrathecal injection of the conditioned medium from bone marrow stromal cells on spinal cord injury in rats. J Neurotrauma. 2018; 35: 521-532.

46. Liu W, Wang $Y$, Gong F, Rong $Y$, Luo $Y$, Tang $P$, et al. Exosomes derived from bone mesenchymal stem cells repair traumatic spinal cord injury by suppressing the activation of A1 neurotoxic reactive astrocytes. J Neurotrauma. 2019; 36: 469-484.

47. Rong YL, Liu W, Wang JX, Fan J, Luo Y, Li L, et al. Neural stem cell-derived small extracellular vesicles attenuate apoptosis and neuroinflammation after traumatic spinal cord injury by activating autophagy. Cell Death Dis. 2019; 10: 340.

48. Ruppert KA, Nguyen TT, Prabhakara KS, Toledano Furman NE, Srivastava AK, Harting MT, et al. Human mesenchymal stromal cell-derived extracellular vesicles modify microglial response and improve clinical outcomes in experimental spinal cord injury. Sci Rep. 2018; 8: 480. 
49. Tsai MJ, Liou DY, Lin YR, Weng CF, Huang MC, Huang WC, et al. Attenuating spinal cord injury by conditioned medium from bone marrow mesenchymal stem cells. J Clin Med. 2018; 8: 23.

50. Wang L, Pei S, Han L, Guo B, Li Y, Duan R, et al. Mesenchymal stem cell-derived exosomes reduce $A 1$ astrocytes via downregulation of phosphorylated NFKB P65 subunit in spinal cord injury. Cell Physiol Biochem. 2018; 50: 1535-1559.

51. Zhou X, Chu X, Yuan H, Qiu J, Zhao C, Xin D, et al. Mesenchymal stem cell derived EVs mediate neuroprotection after spinal cord injury in rats via the microRNA-21-5p/FasL gene axis. Biomed Pharmacother. 2019; 115: 108818.

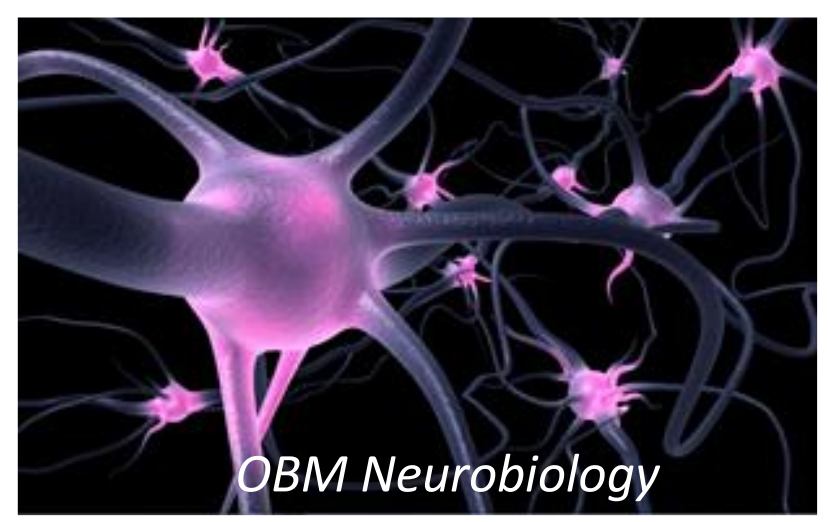

Enjoy OBM Neurobiology by:

1. Submitting a manuscript

2. Joining volunteer reviewer bank

3. Joining Editorial Board

4. Guest editing a special issue

For more details, please visit:

http://www.lidsen.com/journals/neurobiology 